\section{Inhibition of Saccharomyces cerevisiae Pdr5p by a natural compound extracted from Brazilian Red Propolis}

\author{
Cinzia Lotti, ${ }^{1}$ Gabriellen M. Migliani de Castro, ${ }^{2,3}$ Leandro F. \\ Reis de Sá, ${ }^{2}$ Beatriz dos Anjos Fonseca Sampaio da Silva, ${ }^{2,3}$ Ana \\ Claudia Tessis, ${ }^{3}$ Anna L. Piccinelli, ${ }^{1}$ Luca Rastrelli, ${ }^{1}$ Antonio \\ Ferreira-Pereira ${ }^{*}, 2$
}

${ }^{1}$ Dipartimento di Scienze Farmaceutiche, Università degli Studi di Salerno, Italy, ${ }^{2}$ Laboratório de Bioquímica Microbiana, Departamento de Microbiologia Gerall IMPPG/CCS, Universidade Federal do Rio de Janeiro, Brazil, ${ }^{3}$ Instituto Federal do Rio de Janeiro, Brazil.
Revista Brasileira de Farmacognosia Brazilian Journal of Pharmacognosy 21(5): 901-907, Sep./Oct. 2011

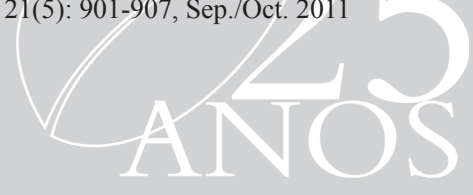

Article

Received 28 Dec 2010

Accepted 17 May 2011

Available online 12 Aug 2011

\begin{abstract}
Multidrug resistance of cancer cells and pathogenic microorganisms leading to the treatment failure of some forms of cancer or life-threatening bacterial or fungal infections is often caused by the overexpression of multidrug efflux pumps belonging to the ATP-binding cassette transporters superfamily. The multidrug resistance of fungal cells often involves the overexpression of efflux pumps belonging to the pleiotropic drug resistance (PDR) family of ABC transporters. Possibly the best-studied fungal PDR transporter is the multidrug resistance transporter Pdr5p of Saccharomyces cerevisiae. Some research groups have been searching for new inhibitors of these efflux pumps in order to alleviate resistance. Natural products are a great source for the discovery of new compounds with biological activity. Propolis is a complex resinous material collected by honeybees from exudates and buds of certain plant sources and this material is thought to serve as a defense substance for bee hives. Propolis is widely used in traditional medicine and is reported to have a broad spectrum of pharmacological properties. Literature reported some biological functionalities of propolis, such as antibacterial, antiviral, fungicidal, antiinflammatory and anti-carcinogenic activities. The chemical composition of propolis is qualitatively and quantitatively variable. Components isolated from methanolic extract of red Brazilian propolis (Alagoas, Northeast of Brazil) are isoflavonoids (including pterocarpans, isoflavans, isoflavones), flavanones and polyprenylated benzophenones. In this work we demonstrated the effects of five different isolated compounds on the ATPase activity of Pdr5p. Out of all five substances tested, only BRP-1 was able to completely abolish the enzymatic activity while others worked as positive modulators of the enzyme activity. BRP-1also inhibited the efflux of Rhodamine $6 \mathrm{G}$ from yeast cells overexpressing Pdr5p. Taken together, these results demonstrate that Brazilian propolis could be a source of promising compounds that can alleviate the MDR phenomenon, particularly in some fungi, where it could be used as an adjuvant for the treatment with azoles.
\end{abstract}


resistance (MDR) poses a severe and increasing public health threat, since new pharmaceutical drugs, especially drugs, show moderate to severe toxicity and/ or side effects (Shukla et al., 2008). Efforts to identify natural products as inhibitors of MDR exporters have the potential to provide a large number of novel drug leads since many of these products have been used for centuries without harmful side effects (Wang et al., 2010).

Propolis is a complex resinous material collected by honeybees (Apis mellifera) from exudates and buds of certain plant sources and this material is thought to serve as defense substance for bee hives (Pietta et al., 2002). It is used to block holes and cracks, to repair combs, to strengthen the thin borders of the comb and for making the entrance of the hive weathertight or easier to defend. Propolis is also used as an "embalming" substance to cover hive invaders which bees have killed but cannot transport out of the hive (Ghisalberti, 1978). The action against microorganisms is an essential characteristic of propolis and it has been used by human beings since ancient times for its pharmaceutical properties. For all types of propolis a broad spectrum of pharmacological properties is reported. Literature reported some biological functionalities of propolis, such as antibacterial and antiviral properties and many other beneficial biological activities including antiinflammatory, antiulcer, local anaesthetic, hepatoprotective, antitumor and immunostimulating properties (Sforcin et al., 2000; Kujumgiev \& Popov, 1996; Banskota et al., 2002; Awale et al., 2008; Marcucci, 1995; Burdok, 1998; Popova et al., 2005). For this reason propolis is widely used as a popular remedy in folk medicine, as a constituent of biocosmetics, health food and today represents an important raw material for many health products and constitute a new interdisciplinary area for research (Matsuda, 1994).

Propolis chemical composition is qualitatively and quantitatively variable, depending on the season, the species of bee, vegetation and the area of collection; in tropical regions, for example, because of the difference in vegetation, the chemical composition of propolis is very different (Lotti et al., 2010). Its colour also varies depending on its botanical source, the most common being dark brown, but red propolis has also been observed in tropical countries such as Brazil and Cuba (Park et al., 2002; Cuesta-Rubio et al., 2007). The major components isolated from methanolic extract of red Brazilian propolis (Alagoas, Northeast of Brazil) are isoflavonoids (including pterocarpans, isoflavans, isoflavones), flavanones and polyprenylated benzophenones (Trusheva, 2006; Awale et al., 2008). This type of Brazilian Red Propolis (BRP) demonstrated a notable antimicrobial activity against Staphylococcus aureus and mutans (Alencar et al., 2007) and cytotoxicity against human pancreatic PANC-1 cancer cell line (Awale et al., 2008). In this work we show that BRP is also able to inhibit the enzymatic activity and the rhodamine $6 \mathrm{G}$ transport of Pdr5p from Saccharomyces cerevisiae. Thus BRP could be a new tool for the study of the mechanism of multidrug resistance and work as a natural compound to reverse this phenotype in yeast.

\section{Materials and Methods}

\section{Plant material}

Samples of raw red propolis produced by Apis mellifera bees were collected in a Northeast region of Brazil (Alagoas State) in the mangrove area. Methanol extract of BRP was obtained by maceration of ground propolis sample with methanol and stored at $5{ }^{\circ} \mathrm{C}$ in the dark.

\section{Standard and samples}

Compounds BRP-1-5 were isolated from Brazilian red propolis sample and identified by spectroscopic methods.

\section{General experimental procedure}

A Bruker DRX-600 NMR spectrometer, operating at $599.19 \mathrm{MHz}$ for ${ }^{1} \mathrm{H}$ and at $150.86 \mathrm{MHz}$ for ${ }^{13} \mathrm{C}$, using the UXNMR software package was used for NMR experiments; chemical shifts are expressed in $\delta$ (parts per million) referring to the solvent peaks $\delta \mathrm{H}$ 3.34 and $\delta \mathrm{C} 49.0$ for $\mathrm{CD}_{3} \mathrm{OD}, \delta \mathrm{H} 7.27$ and $\delta \mathrm{C} 77.0$ for $\mathrm{CDCl}_{3}$; coupling constants, J, are in hertz. Preparative HPLC was performed on a Agilent 1100 series system consisting of a G-1312 binary pump, a G-1328A Rheodyne injector, a G-1322A degasser and a G-1315A photodiode array detector (PDA). TLC analyses were performed with Macherey-Nagel precoated silica gel $60 \mathrm{~F}_{254}$ plates.

\section{Isolation of compounds}

A portion of dry extract was fractionated over Sephadex LH-20 column (100 cm x $5.0 \mathrm{~cm})$ using methanol as elution solvent. Major fractions were purified by HPLC-RI (Waters 590 series pumping system equipped with a Waters R401 refractive index detector) in isocratic elution mode using Luna C8 column (250 x $10 \mathrm{~mm}, 10 \mu \mathrm{m}$ ) (methanol-water 55:45 v/v), both from Phenomenex (Torrance, CA, USA), to yield pure compounds, which were identified as isomeric mixture of guttiferone E and xanthocymol (1) (Gustafson et al., 
1992), 7-O-methylvestitol (2) (Piccinelli et al., 2005), vestitol (3) (Piccinelli, 2005), biochanin A (4) (Chang et al., 1994), and formononetin (Chang et al., 1994) (5), respectively.<smiles>C=CC(CC=C(C)C)CC12CC(CC=C(C)C)CC3(CC=C(C)C)C(O)=C(C(=O)c4ccc(O)c(O)c4)C(=O)C1(CC=C(C)C)C(C)(C)C32</smiles>
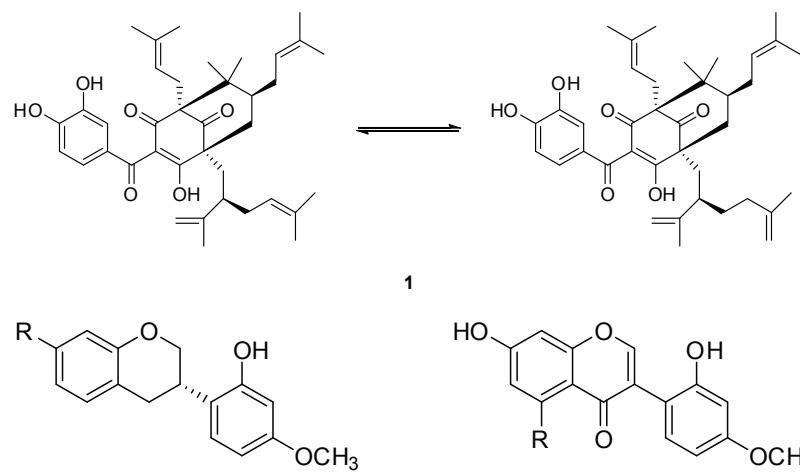
$2 \mathrm{R}=\mathrm{OCH}_{3}$
$3 \mathrm{R}=\mathrm{OH}$<smiles>[R]c1cc(O)cc2occ(-c3ccc(OC)cc3O)c(=O)c12</smiles>
$4 \mathrm{R}=\mathrm{H}$
$5 \mathrm{R}=\mathrm{OH}$

\section{Preparation of plasma membrane}

Plasma membrane fractions were obtained from yeast $S$. cerevisiae mutants AD124567 and AD 1234567 (Decottignies et al., 1998) as previously reported by Rangel et al. (2008). The plasma membranes highly enriched in Pdr5p were stored in liquid nitrogen.

\section{ATPase activity assay}

ATP hydrolysis was determined by incubating Pdr $5 \mathrm{p}$ enriched membranes $(0.013 \mathrm{mg} / \mathrm{mL}$ final concentration) in 96 -well plate at $37^{\circ} \mathrm{C}$ for $60 \mathrm{~min}$ in a reaction medium containing $100 \mathrm{mM}$ Tris- $\mathrm{HCl} \mathrm{pH}$ 7.5, $4 \mathrm{mM} \mathrm{MgCl}, 75 \mathrm{mM} \mathrm{KNO}_{3}, 7.5 \mathrm{mM} \mathrm{NaN}_{3}$ and $0.3 \mathrm{mM}$ ammonium molybdate and $3 \mathrm{mM}$ ATP). The compounds BRP-1-5 were added from stock solution in dimethylsulfoxide up to $5 \% \mathrm{v} / \mathrm{v}$ final concentration. After the incubation period the reaction was stopped with $1 \%$ SDS as described previously (Dulley, 1965). The released inorganic phosphate (Pi) was measured as described previously (Fiske \& Subbarrow, 1925). As control plasma membranes prepared from the PDR5 deleted strain AD1234567 were used. The difference between the ATPase activity of AD124567 membranes and the activity of AD1234567 corresponds to the Pdr5p mediated ATPase activity.

\section{R6G efflux by intact cells}

Rhodamine efflux assays were performed as described previously by Niimi et al. (2004) with minor changes. Briefly, log-phase AD124567 or AD1234567 cells were washed four times with distilled water. The pellet was resuspended in water and incubated for 15 min at $4{ }^{\circ} \mathrm{C}$. Soon after, the cell suspension was centrifuged and the pellet was resuspended in $50 \mathrm{mM}$ 4-2-hydroxyethyl-1-piperazineethanesulfonic acid (HEPES)-NaOH, pH 7.0. To deplete intracellular energy, the cells were incubated for $30 \mathrm{~min}$ a $30{ }^{\circ} \mathrm{C}$ with 2-deoxyglucose (final concentration $5 \mathrm{mM}$ ). Then R6G was added at a final concentration of $15 \mu \mathrm{M}$ and incubated for $30 \mathrm{~min}$ at $30{ }^{\circ} \mathrm{C}$. R6G equilibrated cells were then washed twice with distilled water and resuspended with HEPES buffer to an OD of 10 . Approximately $1 \times 10^{8}$ cells were incubated at $30^{\circ} \mathrm{C}$ with or without BRP-1 and glucose at a final concentration of $0.2 \%$ was added to start the reaction. Glucose free controls were included in all experiments. After 13 min the cells were pelleted and the R6G present in the supernatant was spectrophotometrically quantified using a spectrophotometer (P 582-Micronal- Brazil) at a wavelength of $527 \mathrm{~nm}$.

BRP-1 MIC as a measure of toxicity against S. cerevisiae

The MIC of BRP-1 for Pdr5p-hyperexpressing strain AD124567 and isogenic null mutant AD1234567 was determined by a microdilution method according to Clinical and Laboratory Standards Institute (CLSI; former NCCLS M27-A) with slight modifications using 96-well microtiter plates. Cells in YPD medium were inoculated at $1 \times 10^{4}$ cells per well and incubated at $30{ }^{\circ} \mathrm{C}$ for $48 \mathrm{~h}$ with shaking $(150 \mathrm{rpm})$ in the presence of $50 \mu \mathrm{M}$ of BRP-1. Cell growth was monitored at 600 $\mathrm{nm}$ with a microplate reader (Fluostar Optima, BMG Labtech, Offenburg, Germany).

\section{Intracellular accumulation of $R 6 G$}

This essay was performed as previously described by Sharma et al. (2009) with slight modifications. Yeast cells in the logarithmic phase of growth were washed three times with phosphate-buffered saline $(\mathrm{PBS}) \mathrm{pH} 7,0$. The pellet was resuspended in $\mathrm{PBS}$ and then de-energized by incubating for $45 \mathrm{~min}$ at $30{ }^{\circ} \mathrm{C}$ with 2 -deoxyglucose (final concentration $5 \mathrm{mM}$ ) and dinitrophenol (final concentration $5 \mathrm{mM}$ ). The deenergized cells were pelleted, washed twice $(9000 \mathrm{rpm}$ 2 min) with PBS without glucose, and then resuspended in PBS (with glucose 2\%). The cell suspension was incubated with de BRP-1 (final concentration $100 \mu \mathrm{M}$ ) at $30{ }^{\circ} \mathrm{C}$ by $5 \mathrm{~min}$. Then $\mathrm{R} 6 \mathrm{G}$ was added at a final concentration of $2.2 \mu \mathrm{M}$ and incubated for $30 \mathrm{~min}$ at $30{ }^{\circ} \mathrm{C}$. After washing three times with PBS without glucose (9000 rpm, $2 \mathrm{~min}$ ) the cells were resuspended with the same buffer and their fluorescence visualized using a Nikon Eclipse E400 (Nikon, Japan). 


\section{Statistical analysis}

All experiments were carried out at least three times and the results are expressed as mean \pm SD. Standard deviation and IC50 values were calculated using the computer program Sigma Plot version 10 (SPSS Science Marketing).

\section{Results}

\section{ATPase activity assay}

The effects of BRP-1-5 on the Pdr5p ATPase activity are shown in (Figure 1). It was observed that only BRP-1 was able to inhibit the enzyme activity in a final concentration of $100 \mu \mathrm{M}$. In contrast, BRP-2, 3 and 4 stimulate the enzyme activity while BRP-5 was ineffective. BRP-1 was chosen for the following experiments. The determination of the IC50, value of the Pdr5p ATPase activity for BRP-1 is shown in (Figure 2). The results show that BRP-1 is a very strong inhibitor of the Pdr5p ATPase activity with an apparent IC50 of $1.35 \mu \mathrm{M}$.

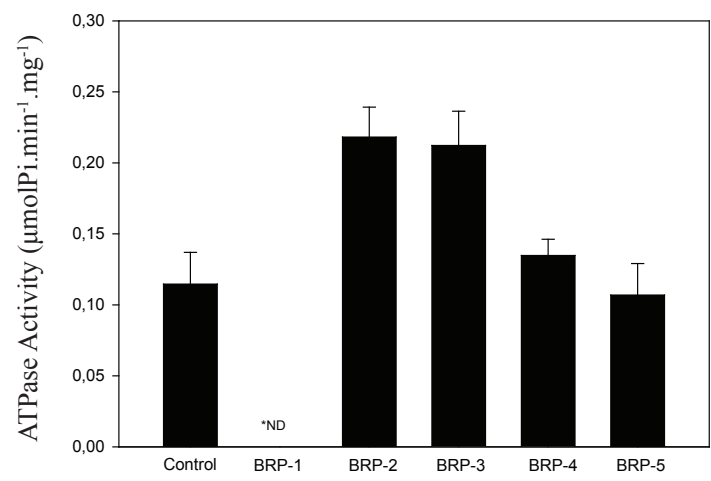

Figure 1. Modulatory effects of BRP compounds on the Pdr5p ATPase activity. Pdr5p-enriched plasma membranes were incubated in the presence of $100 \mu \mathrm{M}$ of BRP-1, BRP-2, BRP-3, BRP-4 and BRP-5. The ATPase activity was measured as described in Materials and Methods. The control bar represents the enzymatic activity in the absence compounds. The data presents means \pm SD of three independent experiments. ${ }^{*} \mathrm{ND}=$ not detected.

\section{Determination of BRP-1 toxicity for $\mathrm{S}$. cerevisiae}

It is known that propolis can be very toxic for bacterial (Alencar et al., 2007) and human cells (Awale et al., 2008). We therefore investigated the toxic effects BRP-1 against $S$. cerevisiae cells. Figure 3 shows that even in the presence of high concentrations of BRP-1 (almost 50 times more than the IC50 value), no toxicity of BRP-1 against yeast cells could be detected (without compound).

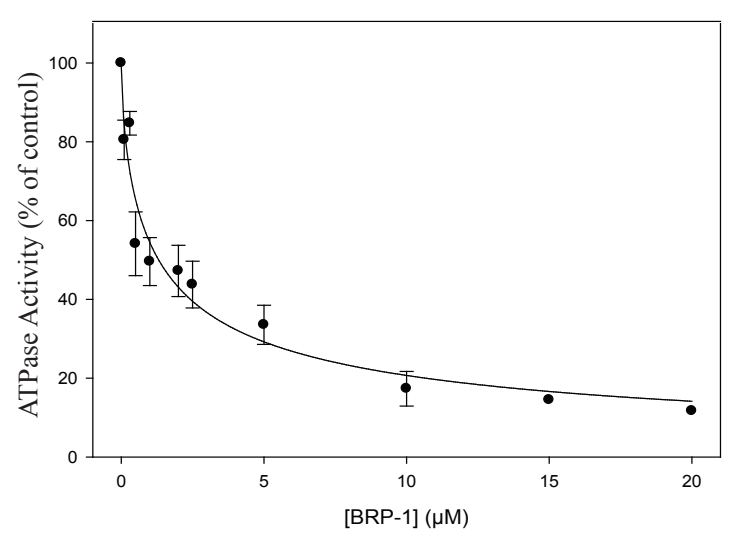

Figure 2. Inhibition of Pdr5p ATPase activity by BRP-1. The enzymatic activity was measured as described in Materials and Methods in the presence of different concentrations of BRP-1 $(0.1$ to $20 \mu \mathrm{M})$. Data represent means \pm SD of three independent experiments. The control represents $100 \%$ of enzymatic activity in the absence of compound.

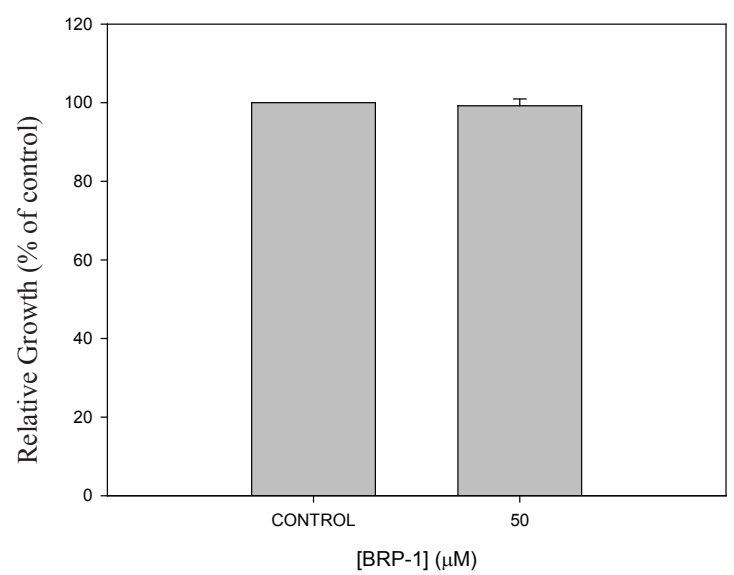

Figure 3. Toxicity of the BRP-1 against AD 124567 strain. The cells were treated with BRP-1 at a final concentration of $50 \mu \mathrm{M}$, as described in Materials and Methods using a 96wells microtiter plate. The control bar corresponds to the $100 \%$ of growth without compound.

\section{Effect of BRP-1 on the intracellular accumulation and the efflux of $R 6 G$}

The effects of BRP-1 on the accumulation and retention of $\mathrm{R} 6 \mathrm{G}$ was first evaluated by fluorescence microscopy (Figure 4). The Pdr5p-hyperexpressing strain AD124567 treated with BRP-1 (final concentration $100 \mu \mathrm{M}$ ) showed a strong fluorescence, indicating an intracellular accumulation of R6G (Figure 4H), compared to the control in the absence of BRP-1 (Figure 4G). In the presence of $100 \mu \mathrm{M}$ BRP-1, AD124567 cells accumulated R6G at levels comparable with those of cells treated with FK-506 (Figure 4F), a known inhibitor of Pdr5p, similar to AD1234567 cells that do not express Pdr5p at all (Figure 4E). 

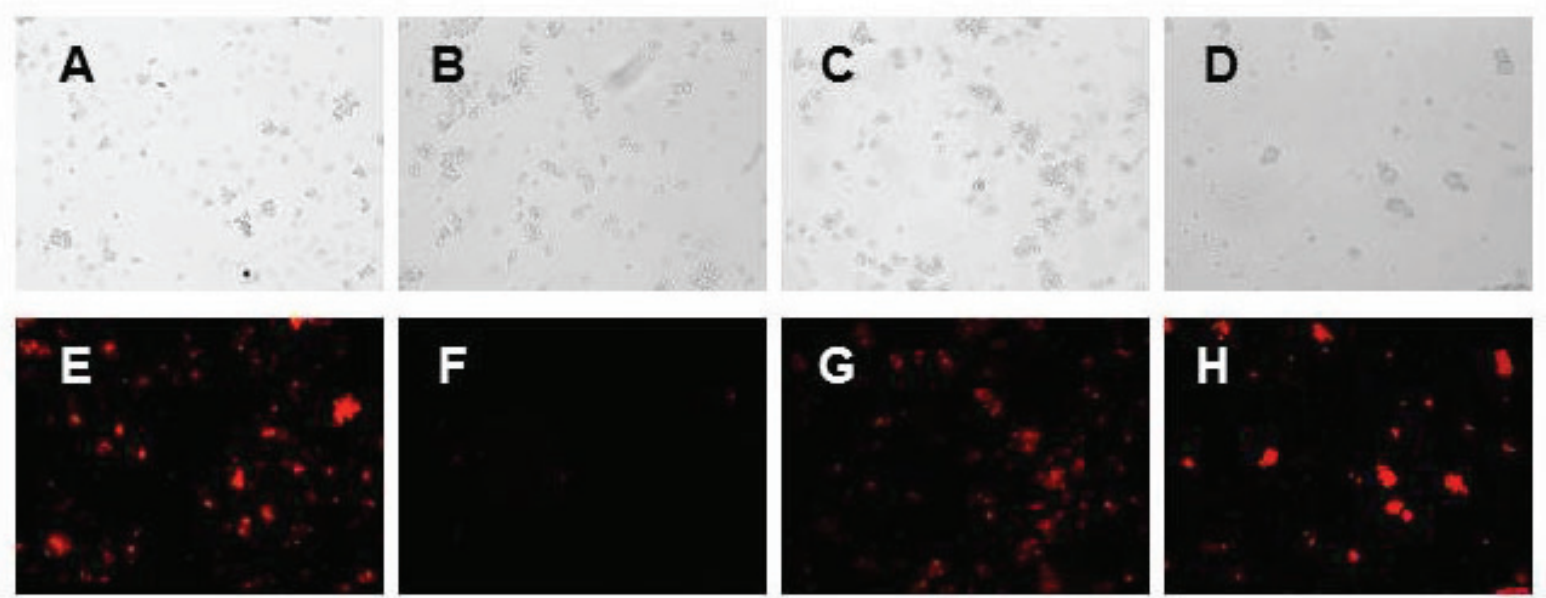

Figure 4. Fluorescence Microscopy of R6G intracellular accumulation of R6G induced by BRP-1. Upper panels (A-D) show the phase contrast microscopy and lower panels (E-H) depict fluorescence of the cells. (A) and (E) show the strain AD1234567 (negative control); (B) and (F) show the Pdr5p-overepressing strain (AD124567); (C) and (G) show the strain AD124567 incubated $2 \mathrm{~h}$ with FK506 (10 $\mu \mathrm{M})$; (D) and (H) AD124567 incubated 2 h with $100 \mu \mathrm{M}$ BRP-1.

In order to quantify the inhibition of energydependent R6G efflux by BRP-1 the R6G efflux of the strain AD124567 was measured at different concentrations of BRP-1 (Figure 5). BRP-1 caused a dose-dependent inhibition of the glucose-dependent R6G efflux, with $80 \%$ of inhibition achieved at 100 $\mu \mathrm{M}$. This inhibition by BRP-1 was very strong with an IC50 value similar to the IC50 value measured for the inhibition of the $(8.14 \mu \mathrm{M})$.

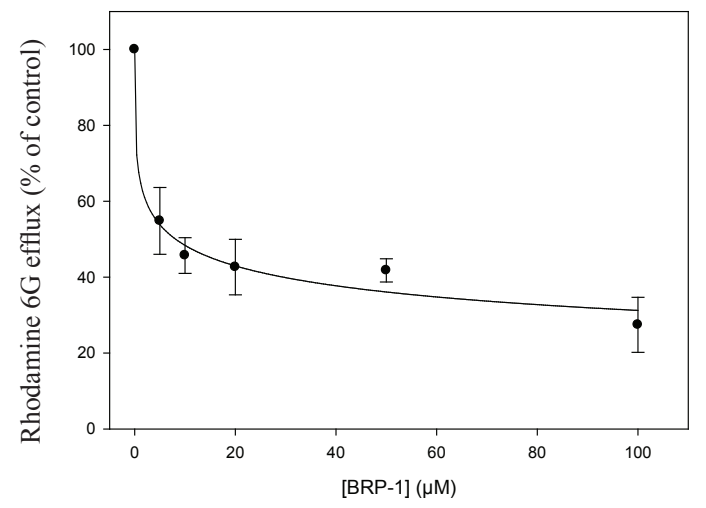

Figure 5. Inhibition of R6G efflux in strain AD124567 by BRP-1. The efflux of R6G was measured after incubation of the cells in the presence of different concentrations of BRP-1 as described in the Material and Methods. Data represent means $\pm \mathrm{SD}$ of three independent experiments.

\section{Discussion}

Resistance to chemotherapy is a clinical problem in patients with infectious diseases caused by prokaryotic or eukaryotic cells. This problem is currently of growing significance with increasing numbers of HIV patients, who are highly susceptible to opportunistic fungal infections due to their severely compromised immune system. MDR caused by the overexpression of multidrug efflux pumps frequently develops in such patients due to their long-lasting prophylactic treatment regimes, but similar MDR can also be observed in some plant and animal pathogens and even in human tumour cells. Therefore, it is necessary to develop strategies that can reverse this drug resistance and natural compounds could be a valuable source material to find potent inhibitors of these multidrug efflux pumps.

In this work, we investigated five different compounds isolated from Brazilian red propolis type, which were purified, identified and named as: guttiferone E/xanthocymol (1); 7-O-methylvestitol (2); vestitol (3); biochanin A (4) and formononetin (5). From those five compounds 1 showed a strong inhibition (Figure 1) of the enzymatic activity of the multidrug efflux transporter Pdr5p of $S$. cerevisiae. Its IC50 value was lower than those reported for other natural compounds such as enniatin (Ivnitski-Steele et al., 2009) or curcumin (Sharma et al., 2009) two well-known inhibitors of multidrug efflux transporters. BRP-1 was not toxic to the yeast cells used in this work even at high concentrations $(50 \mu \mathrm{M})$. Purified BRP-1 $(100 \mu \mathrm{M})$, effectively inhibited the efflux of R6G of Pdr5p overexpressing cells and was able to inhibit the Pdr5p ATPase activity at similar concentrations in vitro (Figure $4 \mathrm{H}$ ). These results suggest that BRP-1 acts as an inhibitor of the Pdr5p ATPase activity by 
blocking its transport mechanism as the R6G efflux of whole cells was effectively inhibited by BRP-1 at low concentrations.

Thus BRP-1 is a very promising natural compound that can effectively inhibit multidrug efflux transporters such as Pdr5p and can be used to reverse MDR of cells overexpressing these efflux pumps function. We expect these results to aid the understanding of Pdr5p and possibly many other multidrug efflux pumps opening new perspectives to improve the treatment of infectious diseases in which these transporters cause treatment failure of traditional synthetic drugs.

\section{Acknowledgments}

We thank Dr. Erwin Lamping (Dental School, University of Otago/Dunedin, New Zealand) for a critical review of this manuscript. The present work was supported by Fundação de Amparo à Pesquisa do Estado do Rio de Janeiro, Coordenação de Aperfeiçoamento de Pessoal de Nível Superior (Procad), Conselho Nacional de Desenvolvimento Científico e Tecnológico and Fundação Universitária José Bonifácio.

\section{References}

Alencar SM, Oldoni TLC, Castro ML, Cabral ISR, Costa-Neto CM, Cury JA, Rosalen PL, Ikegaki M 2007. Chemical composition and biological activity of a new type of Brazilian propolis: Red propolis. J Ethnopharmacol 113: 278-283.

Awale S, Li F, Onozuka H, Esumi H, Tezuka Y, Kadota S 2008. Constituents of Brazilian red propolis and their preferential cytotoxic activity against human pancreatic PANC-1 cancer cell line in nutrientdeprived condition. Bioorg Med Chem 16: 181-189.

Banskota AH, Tezuka Y, Kadota S 2001. Recent progress in pharmacological research of propolis. Phytother Res 15: 561-571.

Burdock GA 1998. Review of the biological properties and toxicity of bee propolis. Food Chem Toxicol 36: 347 363

Chang YC, Nair MG, Santell RC, Helferich WG 1994. Microwave-mediated synthesis of anticarcinogenic isoflavones from soybeans. J Agric Food Chem 42: 1869-1871.

Cuesta-Rubio O, Piccinelli AL, Campo Fernandez M, Marquez Hernandez I, Rosado A, Rastrelli L 2007. Chemical characterization of Cuban propolis by HPLC-PDA, HPLC-MS, and NMR: the brown, red, and yellow Cuban varieties of propolis. J Agric Food Chem 55: 7502-7509.

Decottignies A, Kolaczkowski M, Balzi E, Goffeau A 1994. Solubilization and characterization of the overexpressed PDR5 multidrug resistance nucleotide triphosphatase of yeast. J Biol Chem 269: 12797 12803.
Dulley J 1965. Determination of inorganic phosphate in the presence of detergents or protein. Anal Biochem 67: 91-96.

Ernst R, Klemm R, Schmitt L, Kuchler K 2005. Yeast ATPbinding cassette transporters: cellular cleaning pumps. Methods Enzymol 400: 460-484.

Fiske CH, Subbarow YJ 1925. The colorimetric determination of phosphorus. J Biol Chem 66: 375-400.

Ghisalberti EL 1978. Propolis: a review. Bee World 60: 5984.

Gustafson KR, Blunt JW, Munro MHG, Fuller RW, McKee TC, Cardellina JH 1992. The guttiferones, HIV-inhibitory benzophenones from Symphomia globuliferea, Garcinia livingstonei, Garcinia ovalifolia and Clusia rosea. Tetrahedron 48: 10093-10102.

Hawkey PM, Jones AM 2009. The changing epidemiology of resistance. J Antimicrob Chemother 64 Suppl 1: i310 .

Ivnitski-Steele I, Holmes AR, Lamping E, Monk BC, Cannon RD, Sklar LA 2009. Identification of Nile red as a fluorescent substrate of the Candida albicans ATPbinding cassette transporters $\mathrm{Cdr} 1 \mathrm{p}$ and $\mathrm{Cdr} 2 \mathrm{p}$ and the major facilitator superfamily transporter Mdr1p. Anal Biochem 394: 87-91.

Kujumgiev A, Popov S 1996. Antibacterial diterpenic acids of Brazilian propolis. Z Naturforsch 51: 227-280.

Lamping E, Baret PV, Holmes AR, Monk BC, Goffeau A, Cannon RD 2010. Fungal PDR transporters: phylogeny, topology, motifs and function. Fungal Genet Biol 47: 127-142.

Lotti C, Fernandez MC, Piccinelli AL, Cuesta Rubio O, Hernandez IM, De Simone F, Rastrelli L 2010. Chemical constituents of red Mexican propolis; $J$ Agric Food Chem 58: 2209-2213.

Marcucci MC 1995. Propolis: chemical composition, biological properties and therapeutic activity. Apidologie 26: 83-99.

Matsuda S 1994. Propolis - health care food. Foods \& Food Ingred J Jap 160: 64-73.

Morschhäuser J 2010. Regulation of multidrug resistance in pathogenic fungi. Fungal Genet Biol 47: 94-106.

Niimi K, Harding DR, Parshot R, King A, Lun DJ, Decottignies A, Niimi M, Lin S, Cannon RD, Goffeau A, Monk BC 2004. Chemosensitization of fluconazole resistance in Saccharomyces cerevisiae and pathogenic fungi by a D-octapeptide derivative. Antimicrob Agents Ch 48: 1256-1271.

Park YK, Alencar SM, Aguiar CL 2002. Botanical origin and chemical composition of Brazilian propolis. J Agric Food Chem 50: 2502-2506.

Piccinelli AL, Campo M, Cuesta-Rubio O, Marquez I, De Simone F, Rastrelli L 2005. Isoflavonoids isolated from Cuban propolis. J Agric Food Chem 53: 90109016.

Pietta PG, Gardana C, Pietta AM 2002. Analytical methods for quality control of propolis. Fitoterapia 73: 17-20.

Popova M, Silicib S, Kaftanogluc O, Bankova V 2005. Antibacterial activity of Turkish propolis and its qualitative and quantitative chemical composition. Phytomedicine 12: 221-228.

Pradines B, Pages JM, Barbe J 2005. Chemosensitizers in 
drug transport mechanisms involved in protozoan resistance. Curr Drug Targets Infect Disord 4: 411431.

Rangel LP, Abreu LF, Andrade AR, Leitão SG, Leitão GG, Ferreira-Pereira A 2008. Effect of different extracts from the Brazilian Atlantic Forest on the Pdr5p ATPase activity. Rev Bras Farmacogn 18: 30-36.

Sforcin JM, Fernandes A, Lopes CAM, Bankova V, Funari SRC 2000. Seasonal effect on Brazilian propolis antibacterial activity. J Ethnopharmacol 73: 243-249.

Sharma M, Manoharlal R, Shukla S, Puri N, Prasad T, Ambudkar SV, Prasad R 2009. Curcumin modulates efflux mediated by yeast $\mathrm{ABC}$ multidrug transporters and is synergistic with antifungals. Antimicrob Agents Ch 53: 3256-3265.

Shukla S, Wu CP, Ambudkar SV 2008. Development of inhibitors of ATP-binding cassette drug transporters - present status and challenges. Expert Opin Drug Metab Toxicol 4: 205-223.
Trusheva B, Popova M, Bankova V, Simova S, Marcucci MC, Laguna Miorin P, Pasin da Rocha F, Tsvetkova I 2006. Bioactive constituents of Brazilian Red Propolis. eCAM 3: 249-254.

Wang Z, Ravula R, Cao M, Chow M, Huang Y 2010. Transporter-mediated multidrug resistance and its modulation by Chinese medicines and other herbal products. Curr Drug Discov Technol 7: 54-66.

\section{*Correspondence}

Antonio Ferreira-Pereira

Laboratório de Bioquímica Microbiana, Departamento de Microbiologia Geral/IMPPG/CCS, Universidade Federal do Rio de Janeiro

21941902 Rio de Janeiro-RJ, Brazil

apereira@micro.ufrj.br

Tel.: 552125626494

Fax: $552125608344 \# 108$ 